Journal Club

Editor's Note: These short, critical reviews of recent papers in the Journal, written exclusively by graduate students or postdoctoral fellows, are intended to summarize the important findings of the paper and provide additional insight and commentary. For more information on the format and purpose of the Journal Club, please see http://www.jneurosci.org/misc/ifa_features.shtml.

\title{
Induced Pluripotent Stem Cells and Motor Neuron Disease: Toward an Era of Individualized Medicine
}

\author{
Christopher R. Cashman ${ }^{1-3 *}$ and Lorenzo Lazzerini Ospri ${ }^{1 *}$ \\ ${ }^{1}$ Department of Neuroscience, ${ }^{2}$ Department of Neurology, and ${ }^{3}$ Medical Scientist Training Program-MD/PhD Programs, Johns Hopkins University, \\ Baltimore, Maryland 21210 \\ Review of Amoroso et al.
}

Reprogramming of differentiated somatic cells into a pluripotent state (induced pluripotent stem cells, or iPSCs) has opened a veritable treasure trove of opportunities for regenerative medicine and basic research on human diseases. It is now possible to generate iPSCs from human patients suffering from a variety of disorders and to use these cells as starting material for differentiation into diseaseaffected cell types. Cells so obtained can be used for in vitro cellular studies or in vivo transplantation in animal models of disease, with the aim of investigating pathophysiological processes or screening for new treatments. Patient-derived iPSC generation and directed differentiation is especially fruitful for diseases in which access to affected human cells is difficult. Degenerative disorders affecting motor neurons, such as amyotrophic lateral sclerosis (ALS), are prime examples: progress in the molecular study of ALS has been

Received March 11, 2013; revised April 13, 2013; accepted April 16, 2013. ${ }^{*}$ C.R.C. and L.L.O. contributed equally to the work.

C.R.C. thanks Dr. Ahmet Höke for support and insight during graduate training. C.R.C. is also grateful for helpful comments and suggestions by Jacqueline A. Brosnan and Timothy J. Cashman during manuscript preparation. L.L.0. thanks Dr. Paul Worley and Dr. David Linden for support and advice during graduate training. C.R.C. and L.L.O. are also greatly indebted to Dr. Kimberly Christian for critical comments and suggestions.

Correspondence should be addressed to Christopher R. Cashman, Department of Neurology, Neuromuscular Division, John G. Rangos, Sr. Building, 955 N. Wolfe Street, Room 250, Baltimore, MD 21205. E-mail: ccashma1@jhmi.edu.

DOI:10.1523/JNEUROSCI.1062-13.2013

Copyright $\odot 2013$ the authors $\quad 0270-6474 / 13 / 338587-03 \$ 15.00 / 0$ hampered by the inaccessibility of spinal motor neurons from patients. Therefore, it is not surprising that one of the first attempts to derive disease-specific iPSCs focused on ALS (Dimos et al., 2008).

Many challenges remain before iPSC technology can actualize its tremendous potential for the treatment of specific diseases. Perhaps the greatest is to reliably differentiate cells into specific, homogeneous subtypes. This has often proved elusive, with initial differentiation protocols using complex, poorly characterized media (Lee et al., 2007) producing low yields of highly heterogeneous cell populations. In addition, long differentiation protocols, in which the production of mature motor neurons may require 2 months of culture (Dimos et al., 2008), have frustrated attempts to conduct highthroughput screening or large-scale modeling of disease.

A recent paper published in this journal (Amoroso et al., 2013) made significant progress in addressing many of these issues: first, the authors provided a nonviral method to produce motor neurons with relatively high yield and rapidity; second, they characterized and validated a set of markers used to establish cell identity objectively and with a high degree of confidence; finally, they introduced automatized procedures for cell identification and colony selection, minimizing subjective judgment from experimenters.

The differentiation of pluripotent stem cells, including human embryonic stem cells (hESCs) and iPSCs, into spinal motor neurons is a three-stage process, involving neuralization, caudalization, and ventralization. In vitro cell differentiation largely recapitulates signaling that occurs in vivo during embryogenesis. In the embryo, neural fate is initially specified by BMP signal inhibition to form neuroectoderm. From this tissue, the neural tube is ultimately defined through sonic hedgehog $(\mathrm{SHH})$ signaling, a morphogen initially released from the notochord. Higher amounts of sonic hedgehog define more ventral structures, like the ventral horn, within the early neural tube. Retinoic acid (RA) provides cues to further resolve early neurons as caudal (i.e., cervical) structures, as opposed to rostral structures like the hindbrain. Likewise, in a traditional differentiation protocol, hESCs or iPSCs undergo neuralization with high efficiency via $\mathrm{BMP} / \mathrm{TGF}-\beta$ signaling inhibition, while caudalization and ventralization are achieved by directly supplying $\mathrm{SHH}$ and RA. Alternatively, some protocols use integrating viruses to nonspecifically insert the genes that code for certain differentiation factors (often, the downstream effectors of the $\mathrm{SHH}$ or RA signaling pathways) directly into the cell's genome to overexpress these factors and drive the cell into a certain fate. Amoroso et al. (2013) modified the nonviral protocol to include three factors directly applied to the cells: higher concentration RA and two $\mathrm{SHH}$ pathway activators, purmorphamine and the mouse smoothened (a critical compo- 
nent of SHH signaling) agonist SAG. This combination reduced differentiation time to 3 weeks with a motor neuron yield of $50 \%$, comparing favorably with other high-yield differentiation protocols that rely on viral vectors (Hester et al., 2011). Critically, the protocol Amoroso et al. use is virus-free and therefore better suited to translational applications by eliminating the risk of genomic integration and possible critical gene disruption. Clinical applicability of Amoroso's new protocol could be improved by testing it in feeder-free conditions to determine if high yield and rapidity of motor neuron differentiation persist absent feeder cells.

Surprisingly, mouse SAG was more effective in producing human motor neurons than a human-specific smoothened agonist (Amoroso et al., see Fig. $1 A$ ). This might be a concern if mouse SAG produces off-target or noncanonical effects compared with its human counterpart. Given that the authors possess RNA-seq data of the three-factor versus two-factor differentiation protocol, network analysis of upregulated and downregulated genes may provide insight into how SAG facilitates differentiation (Amoroso et al., see Fig. 3 and supplementary information). In particular, if the addition of SAG only increases $\mathrm{SHH}$ signaling, then $\mathrm{SHH}$ effector genes should be more highly expressed in the three-factor differentiated samples than those produced with the two-factor protocol. Alternatively, the critical facilitating factor for fate specification might be a transient upregulation of SHH signaling, or additional pathways activated by SAG, which would cause only transient or minimal increase in $\mathrm{SHH}$ effector genes or more pronounced upregulation of non-SHH effectors in the RNA-seq data, respectively.

After differentiation, a reliable method to determine cell identity is critical for evaluation of the efficacy of the protocol. The authors unequivocally established cell identity with immunocytochemistry and RNA expression analysis in addition to motor neuron reporter activity. In this study, a Hb9:GFP reporter construct was used where GFP expression was under the control of the Hb9 promoter, and, thus, the reporter was active when cells committed to a motor neuron fate. While this construct is a useful reporter for motor neurons, it does not provide information on subtype identity and, at least theoretically, the long half-life of GFP may allow fluorescence to persist while the Hb9 promoter is no longer active (Li et al., 1998). GFP-positive cells were found to express the specific motor neuron markers HB9 and ISL1 by both immunostaining and RNA expression data (Amoroso et al., see Figs. 2 and 3). These markers were previously thought to be always coexpressed, but Amoroso et al. found nonoverlapping expression in approximately half the cells in vitro (Amoroso et al., see Fig. 2). This expression pattern was examined in vivo in human spinal cords at developmental stages when demarcations between lateral (LMC) and medial (MMC) motor columns are well defined (Amoroso et al., see Fig. 2). Expression of the motor neuron markers was region specific, with MMC staining for both HB9 and ISL1, the lateral aspect of LMC expressing only HB9, and the more medial part of LMC expressing only ISL1. Across the length of the spinal cord, however, the relative expression frequency of the two markers was comparable to that observed in vitro. The authors therefore proposed replacing the established criterion for differentiated motor neurons based solely on HB9 expression with a new criterion that involves counting both HB9- and ISL1-expressing cells. This would introduce an overestimation error, because some cells that express ISL1 are not motor neurons, but the authors argue this is a smaller error than the underestimation resulting from counting only HB9-positive cells.

The authors characterized in vivo markers of cell identity down to regional subspecification with, inter alia, FOXP1 immunostaining. This work revealed that the three-factor protocol provides a novel method for obtaining a motor neuron population enriched with FOXP1-positive cells with LMC identity (Amoroso et al., see Fig. $4 E$ ). The authors claim this specific identity is of potential clinical interest, because LMC neurons innervate distal limb muscles and are among the first to die in ALS patients. However, simply replacing LMC cells will likely not increase ALS patient survival, because respiratory failure, not limb paralysis, is the primary cause of mortality. Respiratory neurons are part of the phrenic motor column (PMC) and hypaxial motor column (HMC), which do not stain for FOXP1, instead expressing HOX5 during differentiation and in their mature state (Philippidou et al., 2012). Thus, while the cells generated by Amoroso et al. are largely FOXP1-positive LMC motor neurons, the cells that drive the diaphragm are part of a distinct FOXP1-negative cell population. Nonetheless, while the LMC neurons may not be appropriate for rescue of respiratory neuron death, they may still be useful for disease modeling and pathogenesis. Additionally, whether any motor neuron can substitute for another in the setting of transplant (e.g., whether an LMC neuron could, in fact, substitute for PMC or HMC neurons to maintain respiratory function) is unknown. A recent paper by Lu et al. (2012) showed that rat and human neural stem cells injected into a rat spinal cord injury site engrafted into the tissue and integrated into host circuitry to promote functional recovery. Studies such as this highlight the remarkable flexibility of the nervous system and suggest that exact neuron subtype matching may not be necessary for cell therapy, although it likely remains critical for studies of disease process and neuronal vulnerability.

Amoroso et al. (2013) conclude their discussion of subtype specification by examining the functional properties of the differentiated motor neurons. The authors verified that these neurons have cell-typical spontaneous $\mathrm{Ca}^{2+}$ activity, respond to glutamatergic stimulation, and fire action potentials in response to current injection (Amoroso et al., see Fig. 6). It would be interesting to investigate whether the "accelerated-maturation" cells also display the characteristic plastic electrophysiological properties of mature motor neurons, for example, spike frequency adaptation (Manuel et al., 2009). As for in vivo functionality, the authors' xenotransplantation assay of neurite outgrowth is an encouraging marker of motor neuron viability, but future experiments should include coculture with myotubes as an assay of neuromuscular junction formation and function.

In conclusion, the lasting significance of this study lies not, in our opinion, in the more conspicuous findings of a new protocol producing differentiated motor neurons with a higher yield, a higher speed, and a different cell subidentity with respect to prior protocols, but in the meticulous work of human in vivo characterization and validation of markers for cell identity. A serious problem in the modeling of human disease by use of iPS cells is the extraordinary cell heterogeneity generated by the differentiation process-a confounding source of "noise," whose detrimental impact on scientific analysis cannot be overstated. Surely this can be minimized by controlling the differentiation conditions, for instance by using small-molecule activators instead of complex, poorly defined media, but the availability of validated cell identity markers standing as universal standards for mature cell purification will likely always re- 
main paramount. A corollary benefit will be the possibility of using these markers in automated cell-sorting and counting techniques (such as FACS) - essential developments to standardize procedures and improve comparability between studies in the future. This study takes us a step closer to making iPSC-modeling of disease a solid technology, offering a reliable alternative to the usual mouse genetic models, and, we hope, eventually delivering on its promise of a new era of individualized medicine.

\section{References}

Amoroso MW, Croft GF, Williams DJ, O'Keeffe S, Carrasco MA, Davis AR, Roybon L, Oakley DH, Maniatis T, Henderson CE, Wichterle $\mathrm{H}$ (2013) Accelerated High-Yield Generation of Limb-Innervating Motor Neurons from $\mathrm{Hu}-$ man Stem Cells. J Neurosci 33:574-586. CrossRef Medline
Dimos JT, Rodolfa KT, Niakan KK, Weisenthal LM, Mitsumoto H, Chung W, Croft GF, Saphier G, Leibel R, Goland R, Wichterle H, Henderson CE, Eggan K (2008) Induced pluripotent stem cells generated from patients with ALS can be differentiated into motor neurons. Science 321:1218-1221. CrossRef Medline

Hester ME, Murtha MJ, Song S, Rao M, Miranda CJ, Meyer K, Tian J, Boulting G, Schaffer DV, Zhu MX, Pfaff SL, Gage FH, Kaspar BK (2011) Rapid and efficient generation of functional motor neurons from human pluripotent stem cells using gene delivered transcription factor codes. Mol Ther 19: 1905-1912. CrossRef Medline

Lee H, Shamy GA, Elkabetz Y, Schofield CM, Harrsion NL, Panagiotakos G, Socci ND, Tabar V, Studer L (2007) Directed differentiation and transplantation of human embryonic stem cell-derived motoneurons. Stem Cells 25:1931-1939. CrossRef Medline

Li X, Zhao X, Fang Y, Jiang X, Duong T, Fan C, Huang CC, Kain SR (1998) Generation of destabilized green fluorescent protein as a transcription reporter. J Biol Chem 273: 34970-34975. CrossRef Medline

Lu P, Wang Y, Graham L, McHale K, Gao M, Di Wu, Brock J, Blesch A, Rosenzweig ES, Havton LA, Zheng B, Conner JM, Marsala M, Tuszynski MH (2012) Long-distance growth and connectivity of neural stem cells after severe spinal cord injury. Cell 150:1264-1273. CrossRef Medline

Manuel M, Iglesias C, Donnet M, Leroy F, Heckman CJ, Zytnicki D (2009) Fast kinetics, high-frequency oscillations, and subprimary firing range in adult mouse spinal motoneurons. J Neurosci 29:11246-11256. CrossRef Medline

Philippidou P, Walsh CM, Aubin J, Jeannotte L, Dasen JS (2012) Sustained Hox5 gene activity is required for respiratory motor neuron development. Nat Neuro 15:1636-1644. CrossRef Medline 